\title{
Metodología para el mejoramiento de la eficiencia energética de la envolvente edlilicia del sector salud
}

\author{
Methodology to improving energy efficiency of building envelope \\ of heath sector
}

\section{Resumen}

Emilia Urteneche* emiliaurteneche@iipac.laplata-conicet.gov.ar Santiago Tomás Fondoso-Ossola* santiagofondoso@iipac.laplata-conicet.gov.ar Irene Martini* irenemartini@iipac.laplata-conicet.gov.ar dantebarbero@iipac.laplata-conicet.gov.ar carlosdiscoli@iipac.laplata-conicet.gov.ar

* Instituto de Investigaciones y Políticas del Ambiente Construido (IIPAC, CONICET-UNLP)

\section{T-}

os establecimientos energo-intensivos del sector salud representan un escenario con significativas potencialidades para la aplicación de medidas de eficiencia energética. Se plantea una metodología que permite identificar y cuantificar el consumo energético, reconocer la situación técnicoconstructiva de base de la edilicia construida y analizar las variables de diseño que tienen mayor incidencia en el ahorro energético y la habitabilidad, tanto a nivel global (establecimiento) como diferencial (áreas). Dicha metodología constituye una herramienta para proponer alternativas específicas de mejoramiento por área, orientadas al reciclado de la envolvente edilicia, a partir de técnicas de "diseño pasivo". Se presenta la aplicación de la metodología a un caso de estudio de la ciudad de La Plata, Buenos Aires, Argentina. Asimismo, su instrumentación permite comparar escenarios entre establecimientos de similares características, y/o replicar la metodología a establecimientos de otras regiones a partir de considerar los lineamientos de las zonas bioclimáticas de la Norma IRAM 11603.

Palabras clave: eficiencia energética; subsector salud; reciclado; envolvente edilicia; diseño pasivo.

\section{Abstract:}

The energy-intensive buildings of the health sector represent a scenario with significant potential for the application of energy efficiency measures. A methodology is proposed that allows to identify and quantify energy consumption and the basic technical-constructive situation of the built building and analyse the design variables that have the greatest impact on energy saving and habitability, both globally (building) and differential (areas). This methodology constitutes a tool to propose specific improvement alternatives by area, aimed at recycling the building envelope, based on "passive design" techniques. The application of the methodology to a case study of the city of La Plata, Buenos Aires, Argentina is presented. Likewise, its instrumentation makes it possible to compare scenarios between buildings with similar characteristics, and / or replicate the methodology to buildings in other regions based on considering the guidelines of the bioclimatic zones of the IRAM 11603.

Keywords: energy efficiency; health subsector; retrofit; building envelope; passive design. 


\section{Introducción}

El problema energético global ha llevado a distintos países del mundo a comprometerse para reducir el impacto ambiental generado por el modelo de consumo de los países más ricos. En el informe de los Objetivos de Desarrollo Sostenible (Organización de las Naciones Unidas, 2020), se manifiesta que, si bien existen algunas mejoras relacionadas a la eficiencia energética, resulta necesario incrementar la aplicación de medidas más concretas para alcanzar los resultados esperados para el 2030.

En Argentina, teniendo en cuenta que aproximadamente dos tercios de la energía consumida es generada a partir de fuentes no renovables (CAMMESA, 2020), la reducción del consumo energético es imprescindible, por ejemplo, para aspirar a cumplir metas relacionadas con la reducción en términos de emisiones de gases de efecto invernadero. Asimismo, el uso eficiente de la energía es una condición fundamental para sentar las bases, considerando la futura implementación de sistemas para el aprovechamiento de fuentes renovables. En el año 2013, el Gobierno Nacional en conjunto con el Ministerio de Ciencia y Tecnología, planteó a través de las mesas de implementación del Plan Argentina 2020 la necesidad de establecer lineamientos orientados a diferentes áreas de Ciencia y Tecnología. Entre estas se pueden mencionar las líneas orientadas a investigar sistemas de acumulación de energía. Para ello se realizó una mesa de implementación con la temática específica "Almacenamiento de energía", en la cual se planteó que, ante la gran demanda de energía, la misma se puede minimizar actuando sobre la eficiencia de la envolvente edilicia, revalorizando e incorporando atributos termo-físicos de su materialidad (Discoli, 2014). Se trata de reconocer y recuperar, a partir de pautas de reciclado edilicio y eficiencia energética, la materialidad como una masa de acumulación térmica, que puede aprovecharse como almacenamiento de energía de base para sostener los niveles térmicos de habitabilidad esenciales.

Desde el punto de vista del consumo final de energía en el país, en el año 2018 se observa que el 33\% corresponde a los Sectores Residencial y Comercial-Público (MINEM, 2017). Dentro de este último Sector, el Comercial y Público, se incluye el subsector Salud. Dicho subsector abarca tres tipos de sistemas de salud: la salud pública; las prestaciones a través del sistema de Obras Sociales y la salud privada. Esto da lugar a sistemas de salud escasamente integrados entre sí y fragmentados social y geográficamente (Belló y Becerril-Montekio, 2011; Bitencourt y Monza, 2017). Asimismo, los tres tipos de sistemas (público, de seguridad social y privado) se caracterizan, en general, por contar con edificios energo-intensivos debido a su infraestructura de uso continuo y equipamiento. Las instalaciones hospitalarias requieren suministro energético de forma permanente para su funcionamiento durante las veinticuatro horas de todos los días del año, por lo que la energía representa un importante costo de funcionamiento (Arocas et al., 2019). Una cama hospitalaria consume lo mismo que cuatro viviendas de $100 \mathrm{~m} 2$ (Celis et al., 2019). Si bien en Argentina el contexto institucional y tarifario ha cambiado en el 2020 (debido, en parte, al congelamiento de tarifas e incremento de infraestructura sanitaria de emergencia en el marco de la crisis sanitaria mundial del COVID-19), la relación de usos y proporcionalidades entre sectores en la matriz energética se mantiene en valores similares.

Si bien el subsector Salud posee una participación menor en el consumo final de la energía respecto a otros sectores, este se caracteriza por ser un servicio público de interés social, que denota un evidente déficit de gestión, distribución de recursos, ineficiente uso de la energía y problemas de habitabilidad higrotérmica, especialmente en el área de responsabilidad estatal. Ante esta situación, se advierten desequilibrios entre la necesidad de mantener niveles de habitabilidad razonables, alta operatividad y elevados presupuestos para afrontar las demandas requeridas. Se observa que no existe un seguimiento en los aspectos relacionados a la calidad edilicia o a la eficiencia de los sistemas electromecánicos, que intervienen directamente en la calidad de los servicios prestados (Discoli, 1999). El trabajo realizado por Arocas et al. (2018) propone estrategias en un edificio perteneciente a un hospital de alta complejidad, focalizándose en el mejoramiento de motores, equipamiento e iluminación, mientras que las propuestas para el mejoramiento de la envolvente edilicia son, únicamente, la incorporación de carpinterías con DVH (doble vidriado hermético) y la pintura exterior. Similar es el caso del estudio realizado por Nourdine y Saad (2020), donde los autores buscan identificar los consumos de energía eléctrica críticos en un hospital utilizando un esquema similar al que proporciona la Norma ISO 50001. En cuanto a los manuales y guías para gestores de energía en hospitales, se exponen pautas generales para un desempeño más eficiente sin profundizar en posibles resultados a partir de la implementación de distintas estrategias (VVAA, 2010; Agencia Chilena de Eficiencia Energética, 2012; Organización Panamericana de la Salud, 2018). En este sentido, el abordaje de los aspectos referidos a la eficiencia energética en los edificios energo-intensivos del sector Salud resulta fundamental para comprender su funcionamiento y poder sugerir pautas que permitan lograr ahorros de energía y sus consecuentes efectos asociados. El reciclaje sistematizado de la edilicia existente del sector Salud, con criterios de diseño pasivo, representa una alternativa viable para reducir en forma sustantiva su consumo energético y los gastos presupuestarios, a la vez que se mejoran la habitabilidad, el confort higrotérmico, la calidad de vida y los aspectos ambientales. Asimismo, como en el sector Salud los costos de la energía están integrados en el presupuesto general proporcionado por el Ministerio de Salud, en el caso del ámbito público, los altos valores significan una reducción de presupuesto para fines médicos y de salud. En consecuencia, al reducir la demanda de energía, 
podrían aumentarse los recursos disponibles para los servicios de este u otro sector.

En el país existen aproximadamente 5.300 establecimientos asistenciales con internación, de los cuales alrededor de 1.600 corresponden al sistema de salud pública (Ministerio de salud, 2018). La red edilicia de salud pública de la República Argentina está conformada por una amplia diversidad de establecimientos, jerarquizados según su nivel de complejidad (alta, media y baja) y su área de incumbencia (regional, zonal, subzona y local). En el ámbito de la provincia de Buenos Aires se presenta una situación singular, dado que existe un sistema de salud de alta complejidad concentrado en e área metropolitana y disperso en el resto de la provincia. Esta red presenta una gran diversidad tipológica, tanto en complejidad sanitaria como en diversidad morfológica asociada a sus cortes históricos.

A partir de lo esbozado, se plantea desarrollar una metodología orientada al reciclado integral de la envolvente edilicia a partir de técnicas de diseño pasivo, cuya instrumentación incluya un catálogo de alternativas tecnológicas-constructivas concretas que resuelvan aspectos térmicos, constructivos, económicos y de aplicabilidad.
Para el desarrollo de esta investigación se utilizará como área de estudio, la Micro-región del Gran La Plata (MRGLP), que pertenece a la Región Sanitaria XI. La misma cuenta con una importante infraestructura de diversa complejidad, contenida en un área geográficamente acotada (Figura 1). Cabe aclarar que, si bien el objeto de estudio de la investigación corresponde a los Establecimientos energo-intensivos del sistema público del sector Salud de la MRGLP, los aportes producidos podrían ser aplicados a un universo mayor a partir de considerar los lineamientos de la Norma IRAM 11603.

\section{Métodos}

Para llevar adelante los objetivos propuestos para este trabajo se plantea el desarrollo de una metodología que consta de tres etapas: (i) Diagnóstico del consumo energético del sector Salud en el área en estudio; (ii) Desarrollo de estrategias tecnológicas constructivas para el mejoramiento de la envolvente edilicia existente, a partir de los resultados obtenidos de la aplicación de la metodología MEEP, Módulos Edilicios Energéticos Productivos (Martini, 2010); y (iii) Análisis del ahorro energético potencial del establecimiento a partir de las distintas alternativas tecnológicas propuestas apropiadas

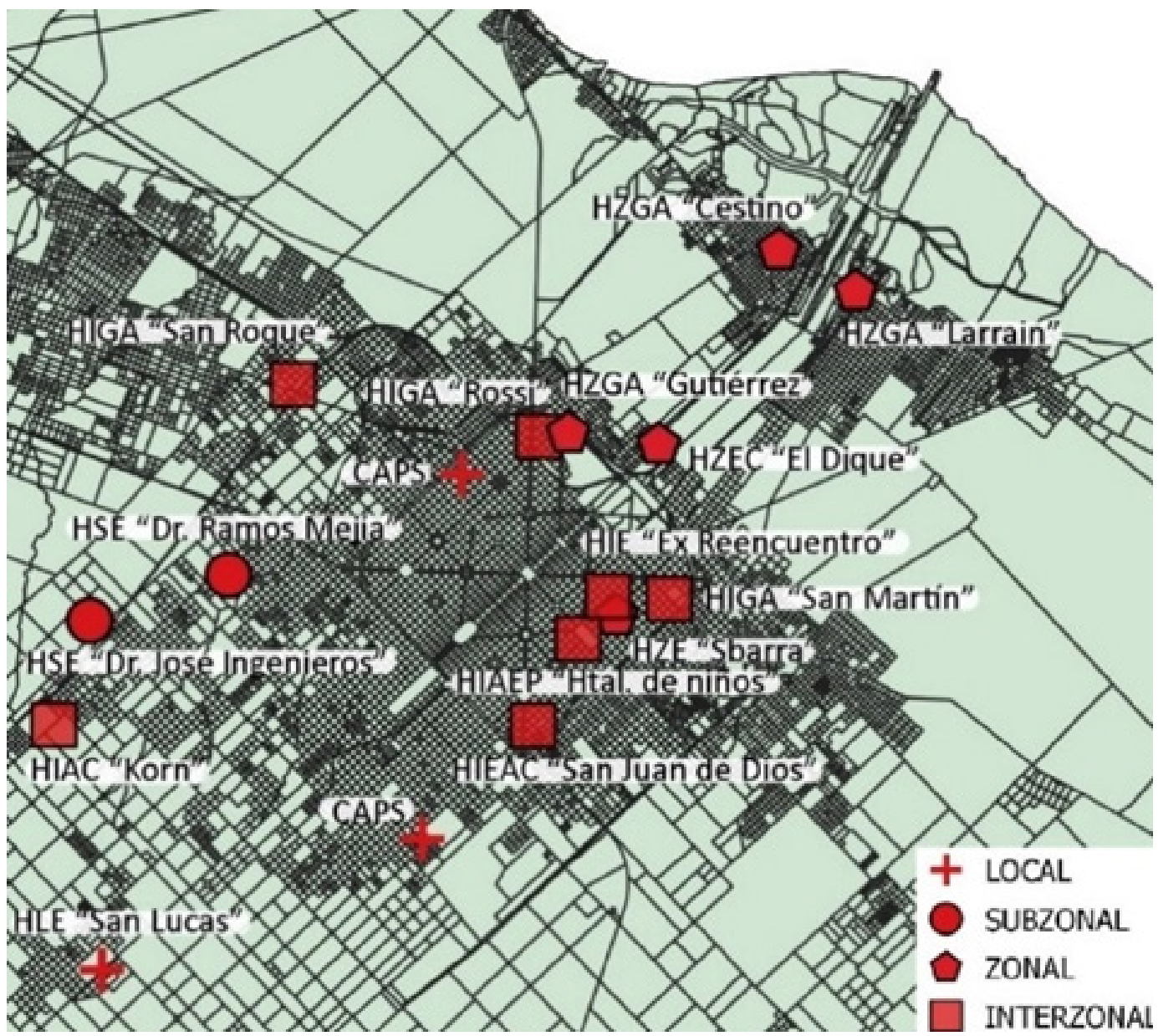

Figura 1: Localización de establecimientos de salud en la MRGLP

Fuente: Elaboración propia 
a las condiciones tecno-económicas de la región. Cada una de las etapas se desarrolla a continuación.

\subsection{Diagnóstico del consumo energético del subsector salud en el área en estudio}

Para el diagnóstico del comportamiento energético en el área de estudio, se analizan y sistematizan los consumos totales de cada establecimiento y su relación con la cantidad de camas. Para ello se obtienen los consumos reales anuales de cada establecimiento, a partir de la lectura de medidores de electricidad y de gas natural y la información productivo-sanitaria brindada por e Ministerio de Salud de la provincia.

Una vez relevado y analizado el comportamiento energético de los establecimientos de salud del sector urbano seleccionado, el siguiente paso consiste en el desarrollo de estrategias de mejoramiento.

\subsection{Desarrollo de estrategias tecnológicas constructivas para el mejoramiento de la envolvente edilicia existente, a partir de la aplicación de la metodología MEEP}

La metodología MEEP utiliza un enfoque analítico ${ }^{1}$ y consiste en modular los comportamientos edilicios energéticos y productivos de la red de salud a partir del análisis particular y detallado. De esta manera, un determinado sector urbano está compuesto por una suma de Redes edilicias. Estas redes representan la suma de Establecimientos o Nodos, integrados por Áreas representativas. Las áreas definidas constituyen un conjunto de Servicios de prestación compuestos por Unidades Funcionales que representan la suma de las necesidades energéticas mínimas de los MEEP base y los MEEP periféricos. LOS MEEP base se definen como diferenciales de análisis de los establecimientos de una red y los MEEP periféricos son aquellos que, a pesar de que en muchos casos no alcanzan la categoría de diferencial de prestación porque representan a los servicios que son complementarios a los producidos por los MEEP base, en algunos casos tienen un peso energético significativo.

De esta manera, es posible identificar y cuantificar los consumos energéticos de cada una de las áreas, y dentro de ellas las variables que tienen mayor incidencia en el Establecimiento, con el objeto de proponer estrategias específicas de mejoramiento de la envolvente que permitan reducir la demanda de energía y el impacto ambiental asociado.

\subsection{Análisis del ahorro energético potencial} del establecimiento a partir de las distintas alternativas tecnológicas propuestas apropiadas a las condiciones tecno-económicas de la región

En esta etapa se utiliza, para evaluar y comparar las diferentes estrategias de reciclado de la envolvente

\footnotetext{
El enfoque analítico consta de dos partes: análisis y síntesis. En la etapa de análisis se divide el problema original en subproblemas más sencillos y se procede a resolver cada uno de ellos. En la etapa de síntesis se combinan los resultados parciales obtenidos en la etapa
} anterior para dar respuesta al problema completo. edilicia, el Índice de eficiencia energético-económica (Rodríguez, 2016), el cual sintetiza la relación entre la optimización del comportamiento térmico y el costo económico de la inversión necesaria para la aplicación de las mejoras tecnológico-constructivas. La ecuación 1 incluye la reducción en el flujo de energía durante una hora por metro cuadrado de envolvente, para una variación de $1^{\circ} \mathrm{C}$, en relación al costo en pesos argentinos $(88,25 \$$ a 1 U\$ al 21/12/20 según cotización del Banco Nación)

$\mathrm{IE}=((\mathrm{KO}-\mathrm{K} 1)) / \mathrm{CI}$

$\mathrm{IE}=$ Índice de eficiencia energético-económica en $\mathrm{W} /$ $\mathrm{m} 2{ }^{\circ} \mathrm{C} \$$

$\mathrm{KO}=$ transmitancia térmica de la envolvente original en $\mathrm{W} /$ $\mathrm{m} 2{ }^{\circ} \mathrm{C}$

$\mathrm{K} 1=$ transmitancia térmica de la propuesta tecnológica constructiva adoptada en $\mathrm{W} / \mathrm{m} 2{ }^{\circ} \mathrm{C}$

$\mathrm{Cll}=$ costo inicial de inversión necesario para la aplicación de las mejoras tecnológico-constructivas en \$.

De esta manera, se calculan los Índices de eficiencia para cada una de las propuestas tecnológico-constructivas para la envolvente horizontal y vertical, en su superficie opaca y transparente. Asimismo, se construye un Índice de eficiencia que considera la intervención de manera simultánea en los muros, cubierta y aberturas, para cada una de las estrategias de reciclado, a fin de facilitar la comparación y elección de las distintas propuestas tecnológico-constructivas. Cuanto más bajo resulta el valor, menos favorable es la situación.

La implementación de una biblioteca de alternativas tecnológicas y de un sistema de valoración permite evaluar rápidamente la viabilidad de las acciones de reciclado, con la posibilidad de su replicación en otras áreas de distintos hospitales con una similar situación original de base, configurando de esta manera una metodología de aplicación de estrategias de reciclado masivo.

\section{Resultados}

\subsection{Diagnóstico del consumo energético del subsector salud en el área en estudio}

Para el diagnóstico del comportamiento energético de la red de salud en la MRGLP, se comenzó por analizar la participación de cada establecimiento en el total de los casos relevados (Tabla 1). El Hospital San Martín registra, al igual que el Hospital Korn, un consumo en Toneladas Equivalentes de Petróleo (TEP) por año significativamente superior al resto de los establecimientos, con una participación del $24 \%$ aproximadamente. Por otra parte, el Hospital ex Reencuentro registra un consumo energético muy por debajo del resto de los establecimientos, con una participación de un 0,2\% del consumo energético total. El resto de los establecimientos tienen un consumo energético entre 40 y 500 TEP, con una participación que oscila entre el $1 \%$ y el $16 \%$.

Con respecto a la cantidad de camas, la Tabla 1 muestra que el Hospital Korn, el Hospital San Martín y el Hospital 
de niños son los que registran un mayor número de camas, con una representatividad de $41,8 \%, 14,4 \%$ y $12,7 \%$ respectivamente en relación al total de la muestra analizada. El Hospital ex Reencuentro es el que presenta el menor número de camas, con un total de 16 y una representatividad de un $0,6 \%$

Si se analiza el consumo energético de cada establecimiento teniendo en cuenta la cantidad de camas, los Hospitales El dique, Rossi, San Martín, el Hospital de niños y el San Juan son los que presentan mayores consumos: 3,63 TEP/cama, 2,81 TEP/cama, 2,28 TEP/ cama, 1,70 TEP/cama y 1,69 TEP/cama, respectivamente. El resto registra un consumo que oscila entre 0,35 TEP/ cama y 1,45 TEP/cama.

Como ejemplo, se seleccionó, el Hospital Zonal Genera de Agudos "Dr. Ricardo Gutiérrez", debido a que presenta una relación TEP/cama dentro del promedio $(1,04)$, por lo que resulta un establecimiento representativo de la muestra analizada. Asimismo, es un hospital de agudos de mediana escala, como la mayoría de los establecimientos del sistema de salud de la provincia de Buenos Aires que se difundieron en mayor medida durante la primera etapa del siglo XX. Además, se dispone del acceso al establecimiento $y$, a partir de relevamientos que se realizaron en el año 2019, se observó la obsolescencia de varios de los equipos de acondicionamiento térmico, lo cual significa un déficit de las condiciones de habitabilidad y confort higrotérmico.

3.2. Desarrollo de estrategias tecnológicas constructivas para el mejoramiento de la envolvente edilicia existente, a partir de la aplicación de la metodología MEEP

En trabajos anteriores (Discoli, 1999; Martini, 2010) se determinó que un establecimiento de salud está compuesto, en general, por las siguientes áreas: Internación, Cirugía, Emergencias, Consultorios externos, Diagnóstico y Tratamiento, Administración, Servicios auxiliares y de Apoyo y Circulaciones. A partir de la aplicación de la metodología MEEP, se analizaron cada una de estas áreas teniendo en cuenta los aportes y pérdidas de energía a partir del estudio de las variables relacionadas a iluminación, equipamiento y climatización (Tabla 2 y Figura 2).

En función de la distribución porcentual planteada, se observa que las áreas de Internación, DiagnósticoTratamiento y Consultorios externos son las que presentan mayores consumos. Asimismo, las áreas de Internación y Consultorios externos son las que requieren mayor consumo de energía para climatización ya que poseen poco equipamiento y requieren bajos

\begin{tabular}{|c|c|c|c|c|c|c|}
\hline \multirow{2}{*}{\multicolumn{2}{|c|}{ Establecimiento }} & \multicolumn{2}{|c|}{ Camas } & \multicolumn{2}{|c|}{ Consumo de energía } & \multirow{2}{*}{$\begin{array}{l}\text { Consumo por cama } \\
\text { TEPaño/cama }\end{array}$} \\
\hline & & Cant. & $\%$ & TEP/año & $\%$ & \\
\hline 1 & HIGA "Gral. San Martín" & 359 & 14,4 & 816,8 & 24,0 & 2,28 \\
\hline 2 & HIAC "Dr. Alejandro Korn" & 1043 & 41,8 & 813,7 & 23,9 & 0,78 \\
\hline 3 & HIAEP "Sor María Ludovica" & 316 & 12,7 & 537,9 & 15,8 & 1,70 \\
\hline 4 & $\begin{array}{l}\text { HIGA Prof. "Dr. Rodolfo } \\
\text { Rossi" }\end{array}$ & 91 & 3,7 & 255,9 & 7,5 & 2,81 \\
\hline 5 & HIGA "San Roque" & 158 & 6,3 & 228,9 & 6,7 & 1,45 \\
\hline 6 & HIEAC "San Juan de Dios" & 133 & 5,3 & 225,0 & 6,6 & 1,69 \\
\hline 7 & HZEC "El Dique" & 54 & 2,2 & 196,0 & 5,8 & 3,63 \\
\hline 8 & HZGA "Dr. Mario V. Larrain" & 87 & 3,5 & 103,0 & 3,0 & 1,18 \\
\hline 9 & $\begin{array}{l}\text { HZGA “Dr. Ricardo Gutié- } \\
\text { rrez” }\end{array}$ & 98 & 4,0 & 101,4 & 3,0 & 1,04 \\
\hline 10 & HGA "Dr. Horacio Cestino" & 56 & 2,3 & 73,0 & 2,2 & 1,30 \\
\hline 11 & HZE "Dr. Noel H. Sbarra” & 83 & 3,3 & 41,2 & 1,2 & 0,50 \\
\hline 12 & HIE "Ex Reencuentro" & 16 & 0,6 & 5,6 & 0,2 & 0,35 \\
\hline & TOTAL & 2494 & 100 & 3398,5 & 100 & 18,71 \\
\hline & Valores medios & 208 & & 283,2 & & 1,56 \\
\hline
\end{tabular}
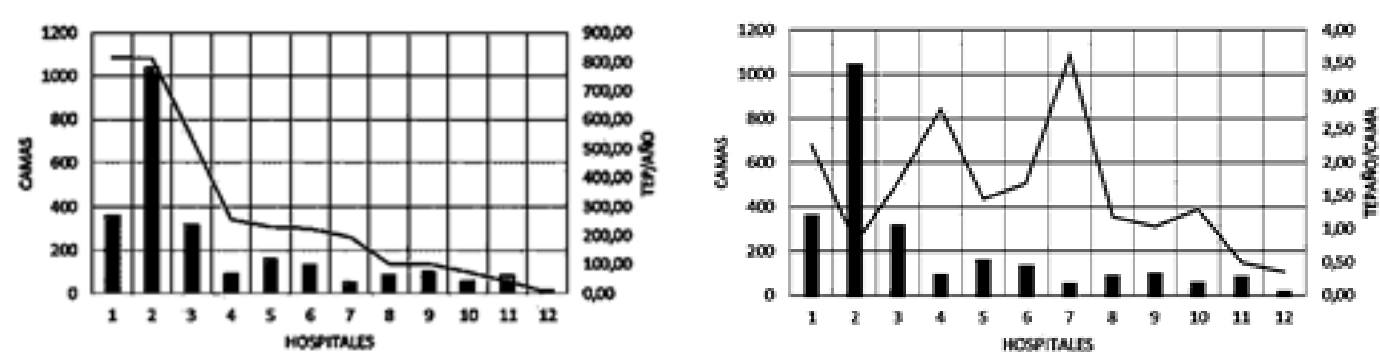

Tabla 1: Cantidad de camas, consumo de energía total y por cama para el universo analizado Fuente: Elaboración propia 


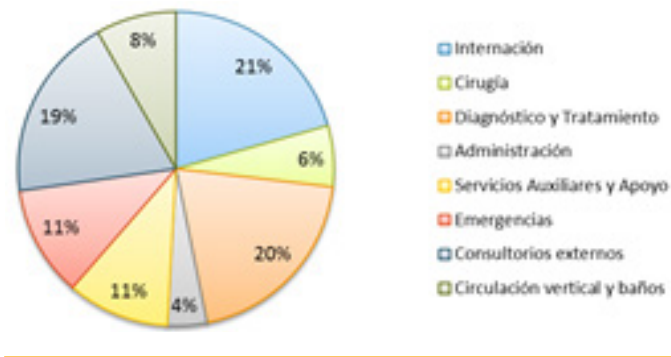

Figura 2: Distribución de los consumos por Áreas de Htal. Gutiérrez

Fuente: Elaboración propia

niveles de iluminación. Esto se debe a que estas áreas se caracterizan por tener mayores superficies vidriadas, generando pérdidas térmicas por renovaciones de aire y por envolvente. En el caso de áreas como Diagnóstico y Tratamiento, por lo general no se requiere energía para calefacción, ya que esta cuenta con importantes aportes de calor por el equipamiento energo-intensivo $y$, habitualmente, no tiene superficie vidriada expuesta.

En este trabajo, se plantea profundizar en la optimización de la energía en climatización del Servicio de Internación
Clínica del Área Internación del Hospital Gutiérrez (Tabla 3). El mismo se encuentra en la planta alta del edificio histórico del Hospital, tiene una superficie de 176,4 m2 y cuenta con habitaciones de 3 camas, orientadas al sureste (Figura 3).

Para obtener la demanda anual del servicio en $\mathrm{kWh} /$ año, se multiplican los valores teóricos de iluminación, equipamiento y climatización de la Tabla 3 por la superficie del Servicio de Internación clínica considerada, teniendo en cuenta su funcionamiento continuo durante todo el año² (Ecuación 2).

En este caso y debido a que solamente se va a optimizar el consumo teórico en climatización, se multiplica el valor teórico de climatización obtenido de la Tabla 3, por la superficie del Servicio y por el período del año que se lo requiere (se consideran 165 días/año), (Ecuación 3). Debido al alto porcentaje que representan los consumos en climatización con respecto al consumo total del establecimiento, es que se propone el desarrollo de estrategias y tecnologías apropiadas para el mejoramiento de la envolvente edilicia existente.

\begin{tabular}{|c|c|c|c|}
\hline HZGA "Gutiérrez" & & CONSUMOS (TEP/año) & \\
\hline ÁREAS & $\begin{array}{c}\text { lluminación + Equipamiento + } \\
\text { Climatización }\end{array}$ & Iluminación + equipamiento & Climatización \\
\hline Internación & 29,84 & 4,61 & 25,23 \\
\hline Cirugía & 9,09 & 2,87 & 6,22 \\
\hline Diagnóstico y Tratamiento & 28,99 & 16,91 & 12,08 \\
\hline Administración & 5,86 & 1,34 & 4,52 \\
\hline Servicios auxiliares & 15,22 & 8,71 & 6,51 \\
\hline Emergencias & 16,52 & 0,44 & 16,08 \\
\hline Consulta externa & 27,53 & 0,73 & 26,80 \\
\hline Circulación vertical y baños & 11,97 & 0,75 & 11,22 \\
\hline TOTAL & 145,02 & 36,35 & 108,67 \\
\hline
\end{tabular}

Tabla 2: Distribución de los consumos en iluminación, equipamiento y climatización por área Fuente: Elaboración propia

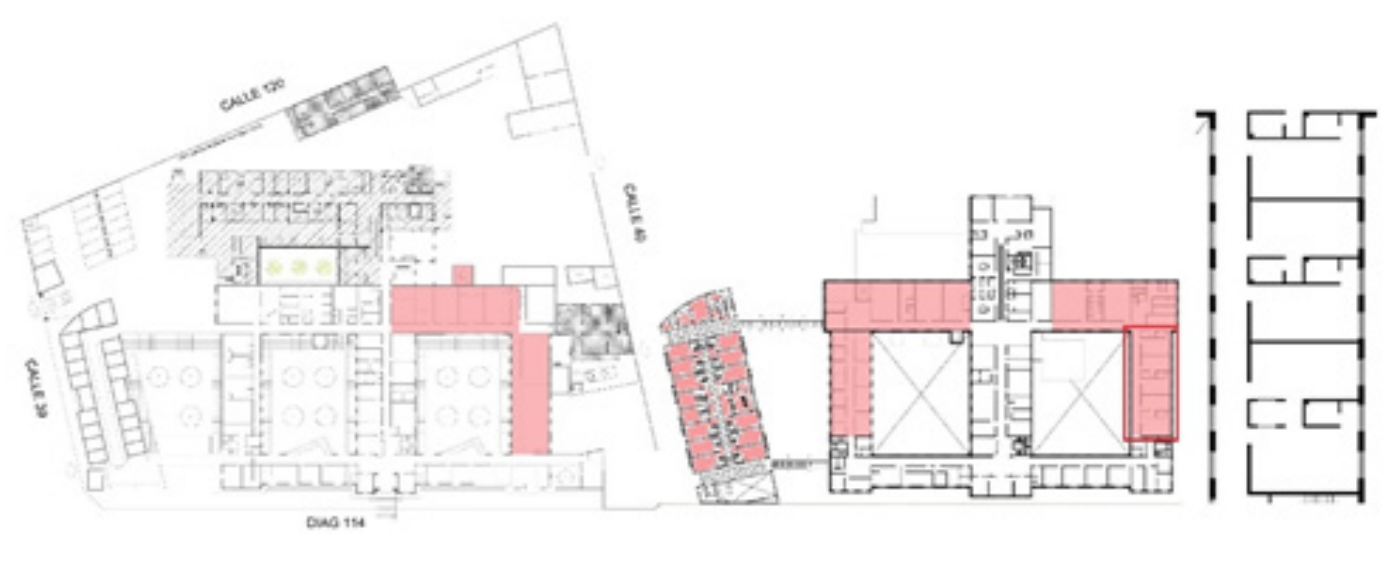

Figura 3: Servicio de Internación Clínica del Área Internación del Hospital Gutiérrez

Fuente: Elaboración propia

\footnotetext{
2 En el caso de iluminación y equipamiento se consideraron 365 días, y para el caso de climatización 165 días/año.
} 


\begin{tabular}{|c|c|c|c|c|c|}
\hline \multicolumn{2}{|c|}{$\begin{array}{c}\text { Hab. } 3 \text { camas } \\
\text { Área: Internación }\end{array}$} & \multirow{2}{*}{$\begin{array}{l}\text { TEÓRICO } \\
\text { Climatiza- } \\
\text { ción }\end{array}$} & \multicolumn{2}{|c|}{ MEEP IN3 } & \multirow{2}{*}{$\begin{array}{c}\text { " ILUMINACICN } \\
\text { kWh/da.m2 } \\
\text { = EQUIPAMIENTO } \\
\text { kWh/diam2 }\end{array}$} \\
\hline Iluminación & Equipamiento & & Tot & Unidad & \\
\hline 0,028 & 0,034 & 0,483 & 0,545 & kWh/día.m2 & kWh/dia.m2 \\
\hline 5,14 & 6,24 & 88,62 & 100 & $\%$ & \\
\hline
\end{tabular}

Tabla 3: Distribución de los consumos en el MEEP teórico del Área Internación: habitación de tres camas (IN3) Fuente: Martini, I. (2010)

$C_{\text {ServiciolN }}=\left\{\left[\left(C_{\text {iluminación }}+C_{\text {equipamiento }}\right)\right.\right.$.período de funcionamiento $]$

$+\left[C_{\text {iclimatización }}\right.$. periodo de funcionamiento $\left.]\right\}$.superficie

$C_{\text {Serviciols }}=\left\{\left[\left(0,028 \mathrm{kWh} /\right.\right.\right.$ día $\cdot \mathrm{m}^{2}+0,034 \mathrm{kWh} /$ dia $\left.\cdot \mathrm{m}^{2}\right) \cdot 365$ días/año $]+$ [0,483 kWh/día.m².165 días/año]\}.176,4 $\mathrm{m}^{2}$

$C_{\text {ServiciolN }}=18.050 \mathrm{kWh} /$ año

$C_{\text {serviciolN }}=C_{\text {climatización }}$. superficie . período de funcionamiento

$C_{\text {servicioln }}=0,483 \mathrm{kWh} /$ día $\cdot \mathrm{m}^{2} \cdot 176,4 \mathrm{~m}^{2} \cdot 165$ días $/ a \tilde{n} o$

$C_{\text {Serviciols }}=14.058 \mathrm{kWh} / \mathrm{año}$

Se plantea disminuir el coeficiente de Transmitancia térmica (K) de la envolvente vertical y horizontal existentes, con el objeto de reducir las pérdidas energéticas. En el caso de aplicación, la envolvente vertical está constituida por muros másicos simples de ladrillo macizo de 0,3 $\mathrm{m}$ de espesor $\left(\mathrm{K}=2,03 \mathrm{~W} / \mathrm{m} 2^{\circ} \mathrm{C}\right)$ y cuenta con ventanas tipo banderola con marco y hoja de chapa doblada y vidrios simples $\left(\mathrm{K}=5,82 \mathrm{~W} / \mathrm{m} 2^{\circ} \mathrm{C}\right)$. La envolvente horizontal consiste en una cubierta inclinada de tejas con ático sin ningún tipo de aislación térmica $\left(\mathrm{K}=2,23 \mathrm{~W} / \mathrm{m} 2^{\circ} \mathrm{C}\right)$. Actualmente, dicho Servicio no cumple con las condiciones de acondicionamiento higrotérmico que exige la Ley 13059-03 de la Provincia de Buenos Aires.

A partir de la situación actual de la edilicia existente, se proponen, a modo de ejemplo, distintas estrategias de intervención de la envolvente:

- Incorporación de aislación térmica desde el exterior El sistema EIFS (Exterior Insulation Finish System) se compone por planchas de poliestireno expandido (EPS), las cuales se adhieren al muro existente por medio de adhesivo (o fijaciones mecánicas) para luego aplicar sobre estas una capa de base coat (mezcla de polímeros acrílicos y cemento Portland). Para darle resistencia mecánica, se refuerza con una malla de fibra de vidrio que cubre toda la superficie de las planchas de EPS. El acabado final se realiza con revestimiento plástico o con pintura exterior. Dicha implementación permite incluir la masa térmica de los muros como estabilizadores de los ambientes, a partir de su inercia térmica. También se incorpora una mejora en el comportamiento térmico de las aberturas mediante la colocación de una cortina de enrollar de aluminio inyectada con poliuretano. En cuanto a la envolvente horizontal, se incorpora aislación térmica exterior con EPS debajo de la cubierta existente. Esta estrategia para cubiertas inclinadas propone una alternativa de aislación mediante la colocación de placas de EPS entre el entablonado o machimbre y la cubierta original de tejas.

- Incorporación de aislamiento térmico desde el interior. Se coloca lana de vidrio con foil de aluminio en su cara interna, protegida mecánicamente con placas de roca de yeso de $12 \mathrm{~mm}$. sobre estructura metálica, las cuales se presentan con una terminación final de pintura de látex para interiores. Dicha intervención excluye la masa térmica de los muros, generando espacios térmicamente más dinámicos, orientados a usos intermitentes con climatizaciones específicas. Además, esta estrategia incorpora una mejora en el comportamiento térmico de las aberturas, reemplazando el vidrio simple original por un doble vidriado hermético (DVH). En la envolvente horizontal, se incorpora en el cielorraso suspendido, aislación interior con lana de vidrio, que tiene un foil de aluminio en una de sus caras, actuando como barrera de vapor.

- Combinación de las dos anteriores: consiste en el reciclado de la superficie opaca de la envolvente vertical a partir de la incorporación de aislación térmica desde el exterior, a través del sistema EIFS. Esta estrategia incorpora una mejora en el comportamiento térmico de las aberturas reemplazando el vidrio simple original 
por un doble vidriado hermético (DVH). En la envolvente horizontal, se incorpora en el cielorraso suspendido, aislación interior con lana de vidrio con barrera de vapor incluida.

Uno de los objetivos del mejoramiento de la envolvente es cumplir con las condiciones de acondicionamiento higrotérmico exigidas por las Normas IRAM y por la Ley 13059-03 de la Provincia de Buenos Aires, manteniendo e inclusive mejorando los niveles de habitabilidad.

\subsection{Análisis del ahorro energético potencial} del establecimiento a partir de las distintas alternativas tecnológicas propuestas apropiadas a las condiciones tecno-económicas de la región

Una vez analizadas las distintas propuestas para e reciclado de la edilicia existente, los resultados de cálculo de las características tecnológicas constructivas de la envolvente para la Situación Real y las resultantes a partir de la aplicación de las medidas de mejoramiento, se sintetizaron en la Tabla 4
En la Tabla 4 se puede observar el mejoramiento en el comportamiento térmico y los costos de inversión para cada una de las propuestas tecnológico-constructivas. La estrategia de reciclado 1, que supone incorporar aislación térmica a la envolvente existente desde el exterior, es la más favorable en cuanto al mejoramiento térmico en relación a los costos de inversión, si se tiene en cuenta la envolvente vertical (muros y aberturas). Sin embargo, se observa que, en el caso de la envolvente horizontal, resulta más conveniente la incorporación de aislación térmica interior, en el cielorraso, ya que esta propuesta permite obtener un mejor comportamiento térmico, con un menor costo de inversión. Si se las analiza de manera global, considerando la intervención simultánea en la envolvente vertical (muros y aberturas) y en la envolvente horizontal (cubierta), se observa que la estrategia de reciclado 2 tiene un mayor Índice de eficiencia energéticoeconómica (IE2=0,00028>IE1=0,00015). Esto se debe al gran porcentaje de superficie expuesta que representa la cubierta. La tercera alternativa (reciclado 3), combinando las mejores situaciones para la envolvente vertical y horizontal de las estrategias de reciclado 1 y 2 , resulta superadora de las anteriores si se tiene en cuenta el ahorro energético y económico (IE3=0,00029).

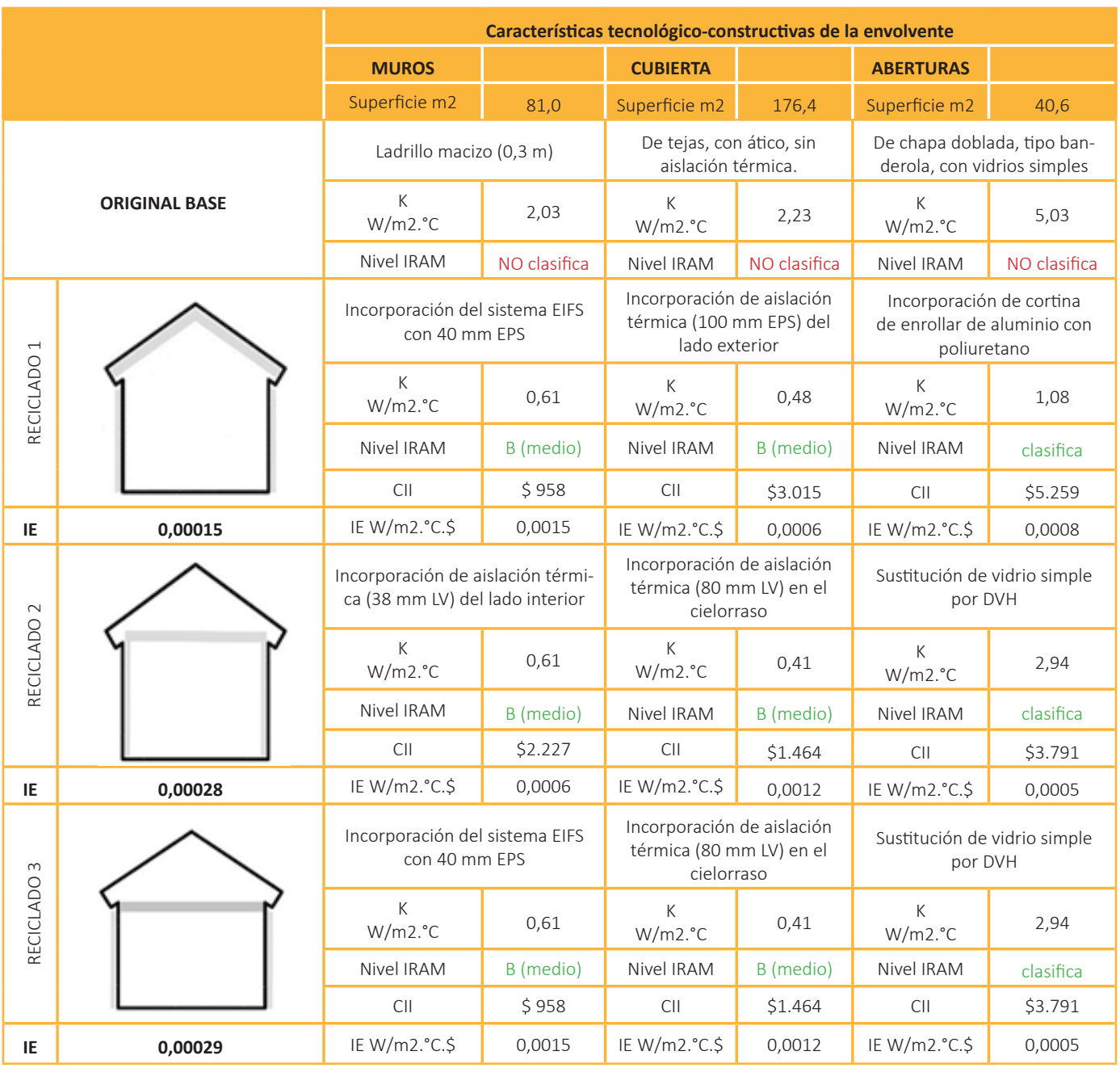


Metodología para el mejoramiento de la eficiencia energética de la envolvente edilicia en el sector salud

En la Tabla 5, se muestra cómo incide la disminución de los coeficientes de transmitancia térmica de la edilicia existente, en la reducción de las pérdidas energéticas por envolvente para el Servicio de Internación Clínica. Al optimizar la envolvente horizontal y vertical, tanto en sus partes opacas, como las transparentes, se obtiene una importante reducción de las pérdidas energéticas. Para las estrategias de reciclado 2 y 3 , si bien el ahorro energético es el mismo, la optimización de la envolvente en el caso 2, requiere un costo de inversión mayor (Tabla 4).

Al disminuir las pérdidas energéticas por envolvente, se reduce la demanda de climatización del Servicio. Ésta se obtiene a partir de la sumatoria de los aportes y pérdidas de energía (Ecuación 4). En este caso, al intervenir en la optimización de la envolvente, las demás variables de la ecuación se mantienen constantes: Cocupación $=0,465$; Ciluminación $=0,021 ; \quad$ Cequipamiento $=0,034 ; \quad$ CGAD $=0,063$; Crenovaciones $=0,901$

$$
C_{\text {climatización }}=\left(C_{\text {ocupación }}+C_{\text {iluminación }}+C_{\text {equipamiento }}+C_{G A D}+C_{\text {renovaciones }}+C_{\text {envolvente }}\right) \cdot\left(k W h / \text { día. } m^{2}\right)
$$

Para la situación original de base resulta:

$$
C_{\text {climatización }}=(0,465+0,021+0,034+0,063+0,901+0,622) \cdot\left(\mathrm{kWh} \text { día } \cdot \mathrm{m}^{2}\right)=0,940 \mathrm{kWh} / \text { día } \cdot \mathrm{m}^{2}
$$

Al reemplazar los valores para la situación optimizada a partir de la estrategia de reciclado 1 resulta:

$$
C_{\text {climatización }}=(0,465+0,021+0,034+0,063+0,901+0,145) \cdot\left(\mathrm{kWh} / \text { día } \cdot \mathrm{m}^{2}\right)=0,463 \mathrm{kWh} \text { día } \cdot \mathrm{m}^{2}
$$

Por último, al reemplazar los valores para las situaciones optimizadas a partir de las estrategias de reciclado 2 y 3 , resulta:

$$
C_{\text {climatización }}=(0,465+0,021+0,034+0,063+0,901+0,197) \cdot\left(\mathrm{kWh} \text { día } \cdot \mathrm{m}^{\wedge} 2\right)=0,515 \mathrm{kWh} \text { día } \cdot \mathrm{m}^{\wedge} 2
$$

La Tabla 6 muestra la demanda de climatización para la situación real y para las situaciones optimizadas, con sus respectivos ahorros. La demanda de climatización para la situación original de base (27.361 kWh/año), calculada a partir de la Ecuación 4, es significativamente mayor a

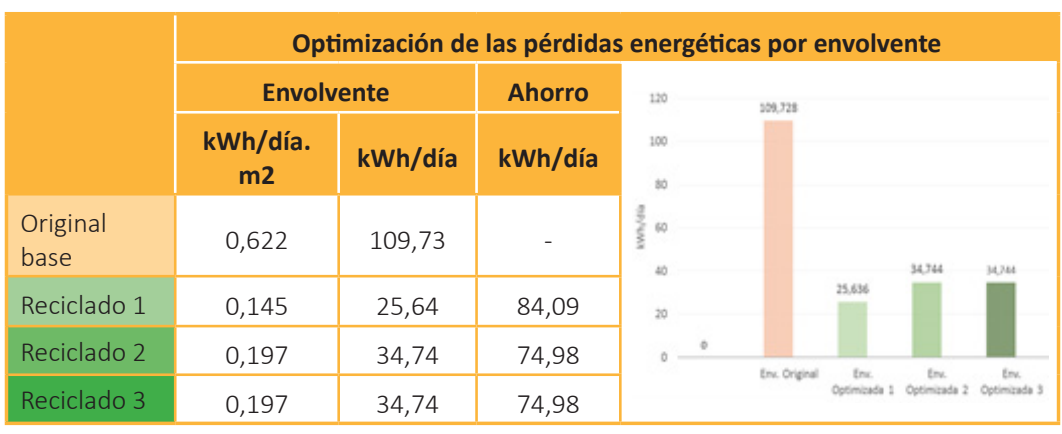

Tabla 5: Cálculo de la energía perdida por envolvente para la situación real y para las situaciones optimizadas ${ }^{3}$ Fuente: Elaboración propia

${ }^{3}$ Pérdidas energéticas por envolvente. Cenvolvente en $\left[\mathrm{kWh} / \mathrm{dí} \cdot \mathrm{m}^{2}\right]=$ (Ko.Ao + Kt.At).1/A.t.GDD.FE donde Ko es la transmitancia térmica de la superficie opaca $\left(\mathrm{W} / \mathrm{m}^{2 \circ} \mathrm{C}\right)$, Ao es la superficie opaca $\left(\mathrm{m}^{2}\right)$, Kt es la transmitancia térmica de la superficie transparente $\left(\mathrm{W} / \mathrm{m}^{2 \circ} \mathrm{C}\right)$, At es la superficie transparente $\left(\mathrm{m}^{2}\right)$, A es la superficie $\left(\mathrm{m}^{2}\right)$, t es el tiempo (hs/día), GDD son los grados día/día (para el área de estudio se consideran $\mathrm{GDD}=6^{\circ} \mathrm{C}$ ) y $\mathrm{FE}$ es el factor de exposición.
${ }^{4}$ Valores tarifarios vigentes desde el 6 de Noviembre de 2019 para usuarios residenciales, comerciales, PyMEs y estaciones de GNC, según lo dispuesto por el Ente Nacional Regulador del Gas (ENARGAS) en las resoluciones № 721 (para Camuzzi Gas Pampeana). Recuperado el día 14 de enero de 2021 de http://www.camuzzigas.com/ tarifas-vigentes. 


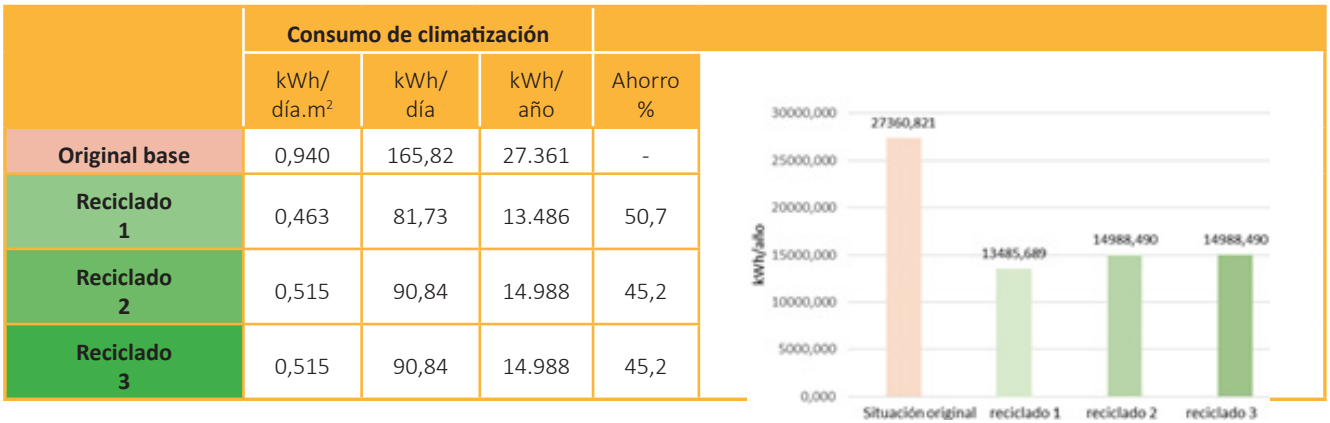

Tabla 6: Comparación de consumos en la situación real y en las situaciones optimizadas Fuente: Elaboración propia

CECanual $=\left[\left(Q /\left(P_{\text {ccombustible.p }}\right)\right) \cdot C C\right]+C F$

CECanual=Costo de Energía en Climatización anual $\mathrm{Q}=$ Carga térmica

$\mathrm{CC}=$ Costo del Combustible. Gas Natural=9,02\$/m3 $\mathrm{CF}=$ Cargo fijo de la factura: $(1.886 \times 12$ meses $)=22.635 \$ /$ año

Pccombustible=Poder calorífico del combustible. Gas Natural=10,7 kWh $/ \mathrm{m} 3$

$\mathrm{p}=$ Rendimiento del equipo de calefacción.

Una vez obtenido el costo de energía en climatización en la situación original, el siguiente paso consiste en determinar el costo del combustible utilizado en cada una de las situaciones optimizadas. El costo fijo de la factura puede variar al disminuir la demanda de climatización, ya que se podría recontratar una potencia menor. Con la aplicación de la estrategia de reciclado 1, el consumo de energía por climatización se reduciría a 13.486 kWh/ año y el costo anual del combustible obtenido resulta e siguiente:

$$
\begin{gathered}
\text { CECanual }=\left[\left(\frac{13.486 \frac{k W h}{a \tilde{n} o}}{10,7 \frac{k W h}{m 3} \cdot 0,8}\right) 9,02 \frac{\$}{m^{3}}\right]+22.635 \frac{\$}{a \tilde{o} o} \\
\text { CECanual }=36.841 \$ / \text { año }
\end{gathered}
$$

Con la aplicación de las estrategias de reciclado 2 y 3 el consumo de energía por climatización se reduciría

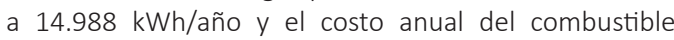
obtenido resulta el siguiente:

$$
\begin{gathered}
\text { CECanual }=\left[\left(\frac{14.988 \frac{k W h}{a \tilde{o} o}}{10,7 \frac{k W h}{m 3} \cdot 0,8}\right) 9,02 \frac{\$}{m^{3}}\right]+22.635 \frac{\$}{a \tilde{n} o} \\
\text { CECanual }=38.424 \$ / \text { año }
\end{gathered}
$$

El paso siguiente a la obtención del costo de la energía para climatización en la situación original y en cada una de las situaciones optimizadas, consiste en analizar el costo de la inversión. Los resultados obtenidos se resumen en la Tabla 7.

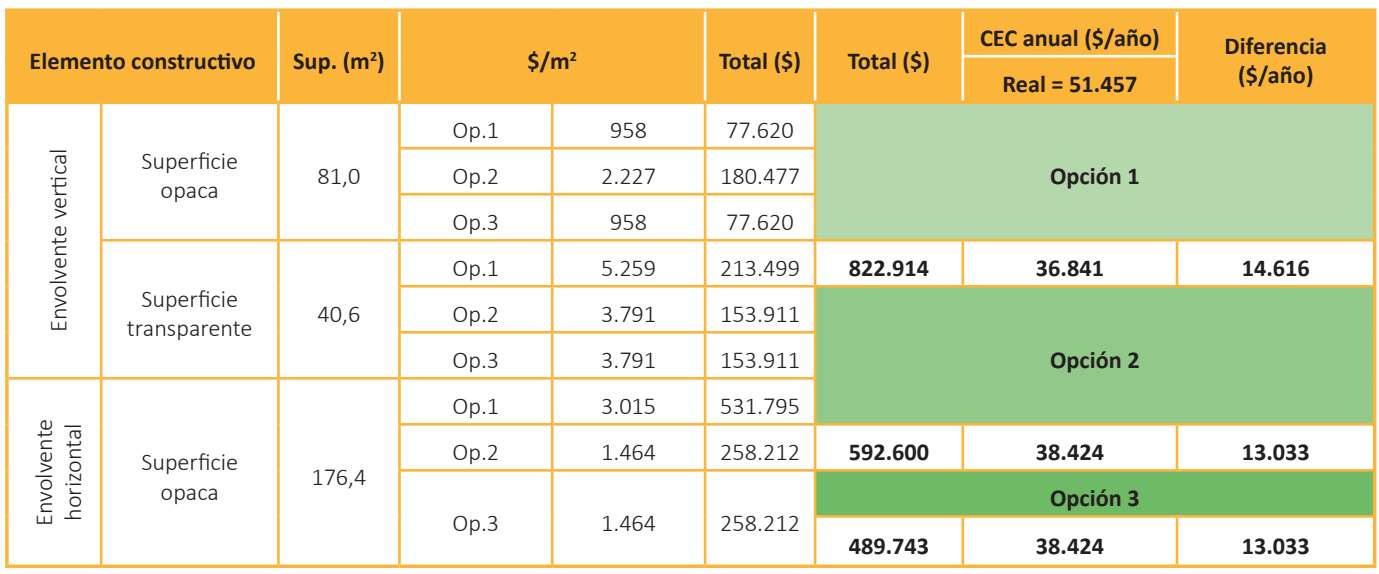

Tabla 7: Resumen de los costos de inversión para las situaciones optimizadas

Fuente: Elaboración propia 
Metodología para el mejoramiento de la eficiencia energética de la envolvente edilicia en el sector salud

En la Tabla 7 se puede observar que, si bien el ahorro en el costo de la energía en climatización anual es apenas mayor con la estrategia de reciclado 1, esta implica costos de inversión significativamente más altos que las estrategias de reciclado 2 y 3 . Para calcular el tiempo que se necesita para amortizar la inversión inicial en cada caso, se utilizó la ecuación $(6)^{5}$

Inversión + CECanualoptimizado $\left[1+\left(\frac{53,8}{100}\right)\right]^{x-1}=$ CECanualoriginal $\left[1+\left(\frac{53,8}{100}\right)\right]^{x-1}[6]$

Inversión $=\left[1+\left(\frac{53,8}{100}\right)\right]^{x-1}[$ CECanualoriginal - CECanualoptimizado $]$

En el caso de la situación optimizada a partir de la aplicación de la estrategia de reciclado 1, despejando la incógnita y aplicando logaritmo natural, resulta:

$822.913,69=(1,538)^{\wedge}(x-1)[51.456,796-36.840,901]$ $x=10,37$

Los resultados obtenidos muestran que se necesitan aproximadamente 10 años para amortizar la inversión inicial.

Para la situación optimizada a partir de la aplicación de la estrategia de reciclado 2 , despejando la incógnita y aplicando logaritmo natural, resulta:

$$
592.599 .92=(1,538)^{\wedge}(x-1)[51.456,796-38.423,933]
$$
$x=9,87$

En este caso, los resultados muestran que se necesita un tiempo menor en relación al caso anterior para amortizar la inversión inicial.

Para la situación optimizada a partir de la aplicación de la estrategia de reciclado 3 , al despejar la incógnita y aplicar logaritmo natural, resulta:

$$
489.743 .23=(1,538)^{\wedge}(x-1)[51.456,796-38.423,933]
$$

Los resultados muestran que se necesita un tiempo aún menor en relación a los casos anteriores para amortizar la inversión inicial.

\section{Conclusiones}

Los hospitales públicos, al ser estructuras energointensivas, representan un escenario con significativas potencialidades para la aplicación de medidas de eficiencia energética, permitiendo reducir los consumos, así como mejorar las condiciones de habitabilidad. Por tratarse de estructuras de uso continuo, con rigurosas condiciones de trabajo, instalaciones complejas $y$ requerimientos estrictos de confort, se encuadran en lo que se denomina "grandes consumidores de energía", requiriendo en consecuencia algún tipo de intervención al respecto. Por otro lado, a pesar de la

\footnotetext{
${ }^{5}$ Se considera un valor de inflación anual fijo según los datos proporcionados por el Instituto Nacional de Estadísticas y Censos (INDEC). Recuperado el día 14 de enero de 2021 de https://www.indec.gob.ar/ uploads/informesdeprensa/ipc_01_20578B3E8357.pdf
}

complejidad funcional de estos edificios, constituyen un espacio demostrativo en el que se pueden aplicar dichas estrategias, estableciendo ejemplos de intervención para gestiones futuras.

El estudio de la red de salud de la MRGLP, que cuenta con una importante infraestructura de diversa complejidad, permite ejemplificar la gran variedad tipológica existente en el subsector, tanto en complejidad sanitaria como en diversidad morfológica-constructiva asociada a sus cortes históricos. Sin embargo, se observa una gran deficiencia como consecuencia del bajo o nulo mantenimiento $y$ del uso ineficiente de las instalaciones. Dicha situación requiere identificar cuáles serían las estrategias más efectivas para lograr un consumo energético eficiente. Para ello, se desarrolló una metodología de abordaje mediante la cual se realizó un análisis y relevamiento del consumo energético para identificar las variables críticas y el peso energético de cada área de prestación dentro de la totalidad del establecimiento de salud. E conocimiento específico de cada nivel de integración desde el punto de vista edilicio, energético, productivo y ambiental, permite determinar la demanda energética, el impacto ambiental y los posibles potenciales de ahorro de cada una de las áreas específicas de los establecimientos de la red de salud. Se identificó para este caso particular y a modo de ejemplo que, intervenir en la envolvente del Área de Internación del Hospital, permite reducir significativamente la demanda de climatización del Área. Se plantean de esta manera, alternativas tecnológicas constructivas con pautas de eficiencia energética específicas para el Servicio de internación clínica a partir del reciclado selectivo de la envolvente edilicia, con técnicas de diseño pasivo, buscando una relación más equilibrada entre la energía utilizada, la eficiencia en el uso y las condiciones de habitabilidad. Esta es una estrategia viable para reducir en forma sustantiva el consumo energético del sector y los gastos presupuestarios consecuentes, mejorando la calidad de vida, el confort higrotérmico y los aspectos ambientales.

Los resultados obtenidos en este estudio permitieron afirmar que, intervenir la envolvente edilicia desde el lado exterior, no solo resulta más práctico desde el punto de vista operativo, ya que el Servicio de Internación podría seguir funcionando casi con total normalidad mientras se realiza la obra, sino que, además, se mejora el comportamiento térmico y la estabilización de los espacios al incluir la inercia térmica de la envolvente. En contraposición, se destaca que, en los casos de edificaciones con valor histórico, se puede intervenir la envolvente edilicia desde el lado interior, mejorando el comportamiento térmico sin alterar la imagen exterior del edificio.

Las estrategias de reducción del consumo a partir del mejoramiento de la envolvente, con períodos de amortización cortos en relación a la vida útil del edificio, implican beneficios que no resultan despreciables; a saber: i. un menor costo operativo durante la vida útil de edificio, y ii. un mejoramiento de la calidad térmica de los ambientes. La eficiencia energética como estrategia busca un equilibrio entre el ahorro de energía y la calidad de vida de los usuarios. 
En síntesis, la metodología desarrollada permitió conocer el consumo energético de cada área en el establecimiento y de cada establecimiento dentro de la red hospitalaria. También constituye una herramienta original para proponer alternativas específicas de mejoramiento a partir de la identificación de las variables críticas de cada área hospitalaria; obtener una visión real para cada situación; y aportar información básica en cuanto a estrategias de optimización, evaluación y valoración de las mismas. Asimismo, dicha metodología permitirá en trabajos futuros comparar escenarios entre establecimientos de similares características, y/o replicar la metodología a establecimientos de otras regiones a partir de considerar los lineamientos de las zonas bioclimáticas de la Norma IRAM 11603.

\section{Agradecimientos}

Este trabajo de investigación contribuye específicamente al siguiente proyecto vigente: i. "Construcción de escenarios energéticos alternativos de desarrollo urbano: implementación de un modelo de simulación integral orientado al uso eficiente de la energía y sustitución de fuentes por renovables." PIP 2018-2020.

Cómo citar este artículo/How to cite this article: Urteneche, E., Fondoso-Ossola, S., Martini, I., Barbero, D. y Discoli, C. (2022). Metodología para el mejoramiento de la eficiencia energética de la envolvente edilicia en el sector salud. Estoa. Revista de la Facultad de Arquitectura y Urbanismo de la Universidad de Cuenca, 11 (21), 141-153. https://doi. org/10.18537/est.v011.n021.a12

\section{Referencias bibliográficas}

Agencia Chilena de Eficiencia Energética. (2012). Manual de Gestor en eficiencia energética. Sector hospitalario. (1era ed.). ACHEE. https://www.guiachileenergia.cl/ agencia-chilena-de-eficiencia-energetica-achee/

Arocas, S. M., Coria Hoffmann, G. D., y Bosc, C. (2018) Estrategias Metodológicas para la Realización de un Diagnóstico de Instalaciones Hospitalarias de un Programa de Ahorro y Eficiencia Energética. Aplicación en el Pabellón de Alta Complejidad del HIGA. En VI Congreso Argentino de Ingeniería Mecánica/ I Congreso Argentino de Ingeniería Ferroviaria. Universidad Nacional de Tucumán.

Arocas, S. M., Coria Hoffman, G., y Bosc, C. (2019). Identificación de indicadores energéticos para el diseño de un programa de ahorro y eficiencia energética en una instalación hospitalaria. $5^{\circ}$ Jornadas ITE, (pp. 770-777). Universidad Nacional de La Plata.

Belló, M. y Becerril-Montekio, V. M. (2011). Sistema de Salud de Argentina. Salud Publica de México, 53(2), 96 108

Bitencourt, F. y Monza, L. (2017). Arquitectura para la salud en América Latina (1a edición). Rio Books.

CAMMESA (2020). Informe anual 2019. Compañía Administradora del Mercado Mayorista Eléctrico Sociedad Anónima. https://portalweb.cammesa.com/MEMNet1/ Pages/Informes\%20por\%20Categor\%C3\%ADa\%20 Publico/Varios/anual.aspx

Celis, F., Echeverría, E., Da Casa, F., Chías, P., y Domínguez, P. (2019). Eficiencia energética y arquitectura hospitalaria en España. Retos y posibilidades. III Encuentro Latinoamericano y Europeo Sobre Edificaciones y Comunidades Sostenibles. Libro de actas (pp. 11931202). Universidad Tecnológica Nacional.

Discoli, C. A. (1999). Control integral y diagnóstico temprano de las redes edilicias de salud. Editorial de la Universidad Nacional de La Plata.

Discoli, C. A. (2014). Informe "Almacenamiento de energía" en la mesa de implementación. IIPAC-FAU-UNLP.

IRAM (1996, 2012). IRAM 11605 Condiciones de habitabilidad en edificios. IRAM 11603 Clasificación bioambiental de la República Argentina. Instituto de Racionalización Argentino de Materiales (IRAM).

Martini, I. (2010). Diagnóstico y mejoramiento de los procesos de gestión edilicia energética productiva en la red de salud [tesis doctoral, Universidad Nacional de Salta].

Nourdine, B., y Saad, A. (2020). Energy Consumption in Hospitals. 2020 International Conference on Electrical and Information Technologies. IEEE. https://doi.org/10.1109/ ICEIT48248.2020.9113177 
Organización de las Naciones Unidas. (2020). Objetivos de desarrollo sostenible. ONU. https://www.un.org/ sustainabledevelopment/es/objetivos-de-desarrollosostenible/

Organización Panamericana de la Salud. (2018) Herramienta para Hospitales Inteligentes. OPS/OMS https://www.paho.org/disasters/dmdocuments/ Hospitales\%20Inteligentes_low.pdf

Rodríguez, L. G. (2016). Instrumentación metodológica para el reciclado masivo de la edilicia residencial orientada a la eficiencia energética [tesis doctoral, Universidad Nacional de Salta].

Secretaría de Energía, Ministerio de Planificación Federal, Inversión pública y servicios (2017). Balance Energético Nacional de la República Argentina, año 2017. Secretaría de Energía. http://datos.minem.gob.ar/ dataset/balances-energeticos

Secretaría de Gobierno de Salud (2018). Indicadores básicos. Argentina 2019. Presidencia de la Nación y Organización Panamericana de la Salud http://www.deis. msal.gov.ar/wp-content/uploads/2019/12/IndicadoresBasicos-2019.pdf

Senado y Cámara de Diputados de la Provincia de Buenos Aires (2003). Ley №13059 de Acondicionamiento higrotérmico. Boletín Oficial de la República Argentina $N^{\circ}$ 24730. Presidencia Argentina.

VVAA. (2010). Guía de Ahorro y Eficiencia Energética en Hospitales. Fundación de la Energía de la Comunidad de Madrid. 